\title{
Summary logarithm of the odds score
}

National Cancer Institute ( $\mathrm{NCl})$

\section{Source}

National Cancer Institute (NCI). Summary logarithm of the odds score.

The summation of all positive pedigree LOD scores (statistical estimates of whether two genetic loci are physically near enough to each other on a particular chromosome that they are likely to be inherited together) at each point in the genome. Also called sumLOD score. 